\title{
Basic Mechanical Parameter Relations of Soft Clay in Pearl River Delta
}

\author{
Zhangming Li*, Na Qi, Zhibin Luo and Weidi Lin
}

Inst. of Geotechnical Engineering, Guangdong University of Technology, Guangzhou, China

\begin{abstract}
Basic parameters relations among CPT parameters, un-drained strength and other mechanical parameters of soft clay are presented based on an elastic-plastic solution for cylindrical cavity expansion for soil investigation in energy engineering. The relation between CPT parameters and shear strength from vane test is also presented based on the result. Thus, the CPT parameters can be determined directly by elastic parameters and shear strength or vane shear parameters and vice versa. That makes it possible to save the high test costs and provide theoretical formulas to avoid some tests which are limited due to the site and/or other condition. Results are compared between the relations and in situ data at a large-scale project in the Pearl River Delta. The results showed consistency between the relation and in situ data.
\end{abstract}

Keywords: Basic mechanical parameters, relations, soft clay, verification.

\section{INTRODUCTION}

The Cone Penetration Test (CPT) is usually used to determine the geo-mechanical properties of soils and to delineate soil stratigraphy and is one of the most widely used and accepted method for soil investigation in energy engineering worldwide, including China. As large-scale construction is conducted in China, especially in the Pearl River delta, a lot of soft ground soil is inevitably encounter, even ultra-soft soil such as soft clay whose natural void ratio is greater than 1.5 and water content greater than the liquid limit. The CPT is a method to determine the basic parameters of soil characteristics, including tip resistance $q_{c}$, side friction $f_{\mathrm{s}}$, friction ratio, $R_{f}\left(=f_{\mathrm{s}} / q_{\mathrm{c}} \times 100 \%\right)$ and specific penetration resistance $P_{s}$ (the quotient of total penetration force and projected area of the probe). These mechanical parameters can be utilized to determine the bearing capacity for foundations, but according to the Building Code in China (Code DBJ 15-60-2008) [1], the CPT can not be used to estimate the un-drained shear strength $S_{u}$ and soil sensitivity $S_{t}$, which are usually obtained from in-situ Vane Shear Test (VST). However, both CPT and VST reflect, to some extent, the soil characteristic of the resistance to shear force and there is reason to believe that certain corresponding relationship exists between CPT and VST parameters. In fact, many researchers have made the link (Chui and Ding, 2004 [2]; Lin, 1994 [3]; Li, 2011 [4]; Robertson and Campanella, 1988 [5]; Robertson and Cabal, 2012 [6]; Yan et al., 2009 [7]; Yang and Xiong, 2010 [8]). However, for saturated ultra-soft clays such as soft clay, related work is very rare.

Based on elastic-plastic analysis of the cavity expansion process and the penetration characteristics of saturated soft

*Address correspondence to this author at the Inst. of Geotechnical Engineering, Guangdong University of Technology, Guangzhou, China;

Tel: +862039322527; Fax: +862039322520; E-mail: ukzmli@163.com clay, this paper is aimed at establishing theoretical relations among VST, CPT and other often used mechanical parameters. They are compared with on-site measured data to evaluate the relationships in order to provide a basis for further understanding of the nature of this kind of ultra-soft soil and also to reduce the amount of tests, to save test cost, and improve test efficiency in practice.

\section{BASIC EQUATION ESTABLISHMENT}

\subsection{Empiric Relations}

A large number of test data were utilized for statistics analysis. To establish certain relations between tip resistance $q_{c}$, side friction $f_{\mathrm{s}}$ and un-drained shear strength of cohesive soils $S_{u}$. The typical statistical fitting equations $(\mathrm{Li}, 2011$ [4], 2014 [9]; Robertson and Campanella, 1988 [5]; Lin, 1994 [3]) can be summed up in a generally linear formula:

$q_{c}=A_{1} S_{u}+B_{1} P_{s}=A_{2} S_{u}+B_{2}$

Where, the statistical fitting coefficient $A_{1}$ and $A_{2}$ are positive constants, while $B_{1}$ and $B_{2}$ could be positive or negative. These coefficients are regarded as constant in most studies except that by Robertson and Campanella (1983). The coefficients were thought to be related to the depth of the measuring point. The two coefficients of the empiric expressions, in short, only the fitting coefficients, have no clear physical-mechanics connotation and cannot be determined by the existing mechanical parameters.

\subsection{Formula Establishment}

The following assumptions were made according to the properties of saturated soft clay and process characteristics of CPT: (1) The cone penetration process can be considered as an elastic-plastic problem in the process of cavity expansion, (2) Use Coulomb strength criterion and assume the value of internal friction angle of saturated soft clay in 
un-drained CPT process, $\varphi=0$; (3) In the cavity expansion process, elastic volume change of the plastic-zone soil is relatively small and can be ignored.

Then the radial expansion pressure $P_{u}$ of the cavity wall (i.e. lateral surface of probe), after a cylindrical cavity expansion, can be obtained upon the above three assumptions (Gong, 1999 [10]).

$$
\begin{aligned}
P_{\mathrm{u}} & =\sigma_{x}^{R_{u}}=c\left(\ln I_{X}+1\right)=c\left[\ln \frac{E}{2(1+v) c}+1\right] \\
\boldsymbol{I}_{x} & =\frac{E}{2(1+v) c}
\end{aligned}
$$

Where $\sigma_{\mathrm{x}}^{R_{u}}$ is the stress at the inside radius of the cylinder after expansion; $c$ is cohesive force; $E$ is the elastic modulus; and $v$ is Poisson ratio.

Take the equilibrium condition of penetration probe:

$$
\pi\left(\frac{D}{2}\right)^{2} q_{c}+\pi D L_{c} f_{s}=\pi\left(\frac{D}{2}\right)^{2} \sigma_{z} q_{c}+\frac{4 L_{c}}{D} f_{s}=\sigma_{z}
$$

Namely

Where $q_{c}$ is Tip resistance; $f_{s}$ is Side friction; $L_{c}$ is Effective friction length of penetration probe, and $D$ is the probe diameter.

Then, $f_{s}$ can be obtained from the radial expansion pressure (multiplied by the friction coefficient):

$$
f_{s}=\mu \sigma_{r}^{R_{u}}=\mu c\left[\ln \frac{E}{2(1+v) c}+1\right]
$$

Where, $\mu$ is the friction coefficient between the penetration probe and the surrounding soil.

The penetration problem is a spatial axisymmetric problem in mechanics. The side and bottom of penetration probe (corresponding to the plane, $r=R_{\mathrm{u}}$ and $z=h$, in the cylindrical coordinates) could be considered as principal plane and according to the balance equation of the axisymmetric problem, that is

$$
\frac{d \sigma_{z}}{d z}=-\gamma z
$$

Solve the equation and obtain the constant according to the boundary conditions, we go

$$
\sigma_{z}=-\gamma^{2} / 2=-h^{2} / 2
$$

Then, the formula on $q_{c}$ can be obtained from the equilibrium conditions of the penetration probe:

$q_{c}=\sigma_{z}-\frac{4 L_{c}}{D} f_{s}=-\frac{h^{2}}{2}-\frac{4 L_{c}}{D} \mu c\left(\ln I_{r}+1\right)$

Where $\gamma$ is natural gravity density of soils; $h$ is the depth of penetration probe.

In Eq. (8), the right side is negative, which indicates that the acting directions of $q_{c}$ and $\sigma_{\mathrm{z}}$ are opposite.
Both single-bridge (the cone together with the outer sleeve, which can only measure a parameter $P_{s}$, used widely in China) and double-bridge (the cone separate with the friction sleeve, which can measure two parameters, i.e. the tip resistance and side friction) penetration probes have the same penetration method and the specific penetration resistance $P_{s}$ is obtained.

$$
p_{s}=q_{c}+m f_{s}
$$

Where, $m$ is the ratio of the effective friction sleeve surface area of single-bridge probe to the double-bridge one. As to the single-bridge and double-bridge probes of the same bottom area, $m$ is the ratio of side effective contact length. For a standard probe with a bottom area of $10 \mathrm{~cm}^{2}$, $m=57 / 179$.

For saturated soft clay, the internal friction angle could be assumed, $\varphi=0$. According to the Coulomb strength criterion, VST shear strength $S_{u}=\tau=c$, put in the Eq. (5) and Eq. (8), then the value of $f_{s}$ and $q_{c}$ could be calculated. These two equations describe the relations between parameters in CPT and VST of saturated soft clay.

Sensitivity coefficient, $S_{t}=S_{u} / S_{u}{ }^{\prime}$, in which $S_{u}$ is undrained shear strength of undisturbed soil, while $S_{u}{ }^{\prime}$ is that of disturbed soil.

Sensitivity coefficient of undisturbed soil can also be estimated by Friction ratio $R_{f}$ of double-bridge. Schmertmann (1978) came up with the relation, $S_{t}=N_{s} / R_{f}$, in which $R_{f}=f_{\mathrm{s}} / q_{\mathrm{c}} \times 100 \%$, then

$$
S_{t}=N_{s} / R_{f}=N_{s} q_{c} / f_{s}
$$

$N_{s}$, as a dimensionless coefficient, were obtained by comparing CPT results with laboratory results by Robertson and Campanella (1988) [5] and the average value is 6. Studies by Rad and Lunne (1986) showed that the $N_{s}$ value changed in the range of $5 \sim 10$, average 7.5. Lunne et al (1997) argued that the value depended on the mineral, OCR and other functions, and no unique value was suitable for all the clay.

The relationship between sensitivity $S_{t}$ from the VST and the parameters of CPT is described in Eq. (10). As to specific penetration resistance $P_{s}$, can be obtained directly from Eq. (9) with $q_{\mathrm{c}}$ and $f_{s}$. So far, four important parameters, including side friction $f_{\mathrm{s}}$, tip resistance $q_{c}$, specific penetration resistance $P_{s}$ and sensitivity coefficient $S_{t}$ can be calculated by Eqs. $(5,8,9,10)$ respectively. The three parameters $f_{\mathrm{s}}, q_{c}$ and $P_{s}$ are the theoretical solutions; while $S_{t}$ is a semi-empirical solution due to it is relative semi-empirical relationship of Schmertmann. Note that these formulas are nonlinear in terms of strength parameters, rather than linear which are expressed by general empirical formulas.

Also, making use of Eq. (5) and Eq. (8), the elastic modulus $\mathrm{E}$ can be calculated by side friction $f_{\mathrm{s}}$ and tip resistance $q_{c}$, respectively:

$$
E=2(1+v) c \cdot e^{\left(\frac{f_{s}}{\mu c}-1\right)} E=2(1+v) c \cdot e^{\frac{D}{4 \mu c L_{c}}\left(q_{c}-\frac{\gamma h}{2}-1\right)}(11 \mathrm{a}, \mathrm{b})
$$

Moreover, the difficulty in determining bearing capacity 
of deep soft soil foundation may be effectively solved when using Eq. (11).

\section{SITE CONDITIONS}

A soft soil site in the Pearl River delta region of China was used to collect in-situ data. The site surface layer is saturated soft clay $11 \mathrm{~m}$ thick, with an average moisture content of $75.0 \%$ and average void ratio of 2.087 .

The strata distribution and physical indicators are shown in Table $\mathbf{1 .}$

\section{TEST METHODS AND EQUIPMENTS}

Before the test, according to geologic condition and working condition of the site the penetration equipment in combination with engineering requirements for the test depth are chosen. Select thrust tonnage of the penetration equipment and prepare a reaction system to ensure the thrust force.

Probes are chosen as shown in Table 2. Relative instruments meet the test requirements.

Calibrations of the probes including strain gauge load cells and pressure transducers were carried out at regular intervals according to relative order and the relevant regulations to verify and ensure the quality of the probes.

VST (Type SB-1Y, measuring range $0 \sim 30 \mathrm{kN}$ ) were carried out at the same plane position of penetration points and conduced below the ground face with a depth of $2 \mathrm{~m}$ or $4 \mathrm{~m}, 6 \mathrm{~m}$ and $8 \mathrm{~m}$.

\section{ANALYSIS AND COMPARISON}

\subsection{In-Site Test Results}

Typical CPT and VST curves are shown in Fig. (1) and Fig. (2) separately. Fig. (1) and Fig. (2) displayed the change in mechanical strength characteristics of this ultra-soft clay before, during and after the improvement. It is clear that the improving effect was obvious in the upper $4 \mathrm{~m}$.

\subsection{Comparison Between Test and Formula Value of Penetration Parameters}

According to soil tests, the relevant parameters were: $S_{u}$ $=8.4 \mathrm{kPa}, E=1.31 \mathrm{MPa}, v=0.35, \gamma=18.5 \mathrm{kN} / \mathrm{m}^{3}, \mu=$ 0.085; Geometric parameters of probes: $L_{\mathrm{c}}=179 \mathrm{~mm}, D=$ $35.7 \mathrm{~mm}$. Took Ns $=6$, which is suggested by Robertson, when calculating sensitivity coefficient $S_{t}$. Calculation depth was $0.5 \sim 8 \mathrm{~m}$. Because of the poor permeability of clay, gravity stress was obtained by calculating water and earth pressures together, and $q_{c}$ or $f_{s}$ was calculated once per $0.1 \mathrm{~m}$ respectively.

Results of comparison between theoretical and test data were shown in Figs. (3 and 4). As shown in the above two figures, the relative laws of the test parameters of CPT were as follows:

(1) In process of penetrating into saturated soft soil, $q_{c}$ grew slowly with $h$, the slope of the calculated curve was not a constant but changed a little; $f_{s}$ kept essentially unchanged with the depth $h$.

(2) The critical depth was not obvious when the probe was penetrated in saturated soft soil. The cause might be ultrasoft clay which is different with other soils. On account of

Table 1. Strata distributing in the test area.

\begin{tabular}{|cll|}
\hline Soil name & $\begin{array}{c}\text { Thickness } \\
\text { / } \mathbf{m}\end{array}$ & \multicolumn{1}{c|}{ Soil description } \\
\hline \hline Artificial hydraulic fill & $0.0 \sim 5.5$ & Distribution is very uneven with, high water content. \\
Soft clay & $3.5 \sim 20.5$ & $\begin{array}{l}\text { Average } 12.0 \mathrm{~m} \text {, plastic flow state. The water content is } 45.8 \% \sim 114 \%, \text { average } 75.0 \% \text {; void ratio of } \\
1.517 \sim 2.992, \text { average } 2.087 .\end{array}$ \\
Silt clay & $0.7 \sim 9.5$ & Alluvial source, plastic state. \\
Sandy clay & $1.0 \sim 12.7$ & Eluvial source, maroon -based, hard plastic, local plastic-like, decomposed granite. \\
Completely decomposed granite & $2.1 \sim 10.5$ & Gray, maroon-based, with a hard core of soil column, easily disintegrating by water. \\
Strongly decomposed granite & $0.7 \sim 13.2$ & Purple, gray, soil cores were folder or chunky rock, soft rock. \\
\hline
\end{tabular}

Table 2. Probe dimensions

\begin{tabular}{|c|c|c|}
\hline Projected area of the Cone $\mathbf{A} / \mathbf{c m}^{2}$ & Probe diameter $\mathbf{D} / \mathbf{m m}$ & \multicolumn{1}{c|}{ Apex angle $\boldsymbol{\alpha} /{ }^{\circ}$} \\
\hline \hline 10 & 35.7 & \multicolumn{2}{|c|}{ Double-bridge probe } \\
\hline Single-bridge probe & Friction sleeve surface area $/ \mathrm{cm}^{2}$ & Friction sleeve length $L_{\mathrm{c}} / \mathrm{mm}$ \\
\hline Effective friction side wall length $L_{l} / \mathrm{mm}$ & 200 & 179 \\
\hline 57 & & 179 \\
\hline
\end{tabular}



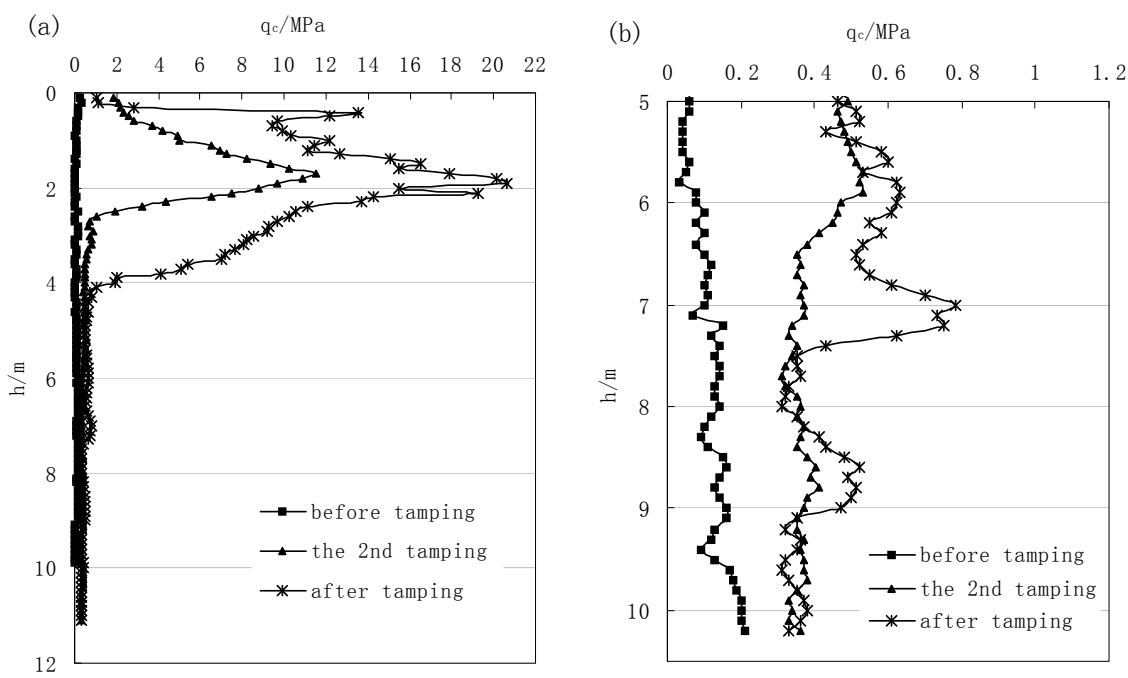

Fig. (1). Relation curve between $q_{c}$ with h (Deepness). a: Total curve, b: Amplificatory curve.
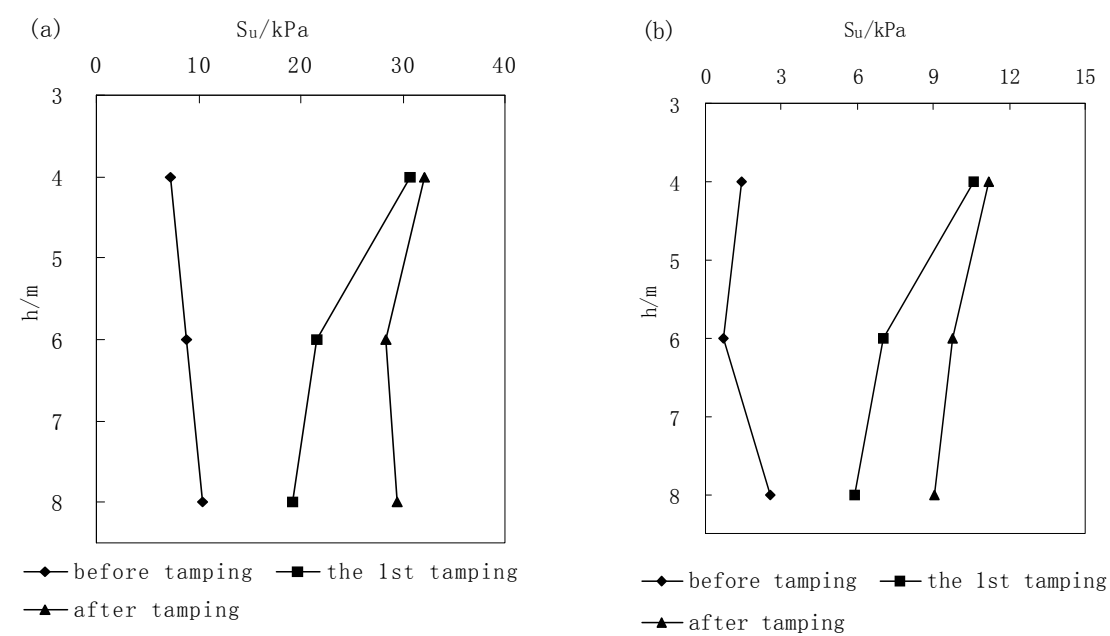

Fig. (2). Typical relation curve of of VST. a: $S_{u} \sim h, b: S_{u}^{\prime} \sim h$.

the small value of friction angle $\varphi$ in saturated soft clay, shear strength and friction showed only minor changes with the increasing depth.

The figure also indicated that the theoretical values were in good consistency with the test ones.

\subsection{Comparison Test Value with Formula Value on $S_{u}$ with Other Parameters}

(1) On the relationship between $q_{c}$ with $S_{u}$

Seen from Fig. (5), the test data shows a positive correlation between $q_{c}$ and $S_{u}$. However, the curve deviates from linearity. The results also indicates that the value of elastic parameters have an effect on results from theoretical calculations. In general, it showed the overall consistency of theoretical and test value.

(2) On the relationship between $f_{s}$ with $S_{u}$

From Fig. (6), the test data shows a general linear trend of $f_{s} \sim S_{u}$. The theoretical value has a better consistency with

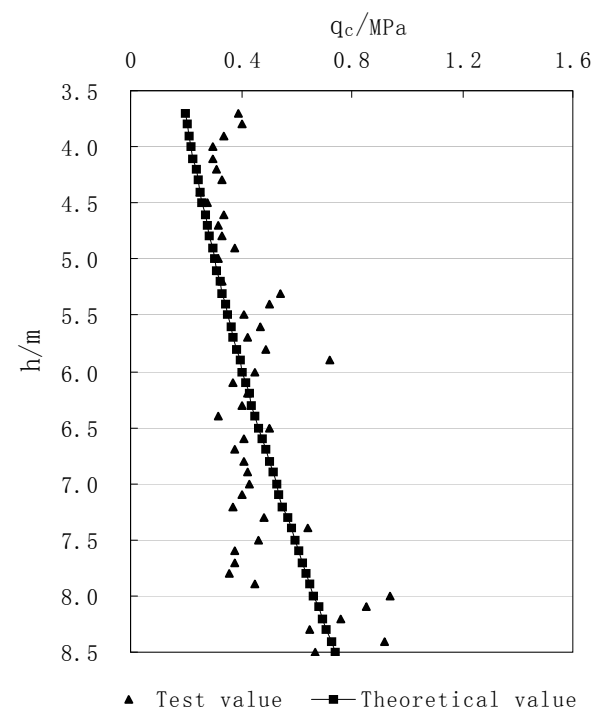

Fig. (3). Relation curve of $q_{c}$. 


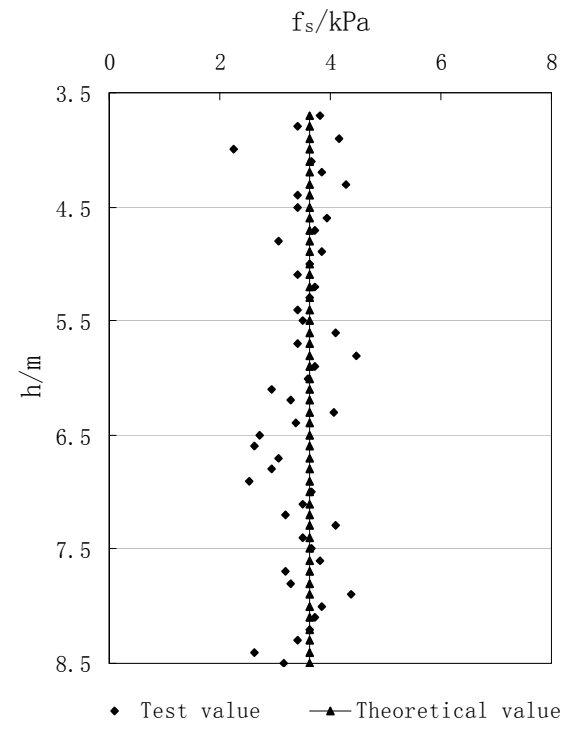

Fig. (4). Relation curve of $f_{s}$.

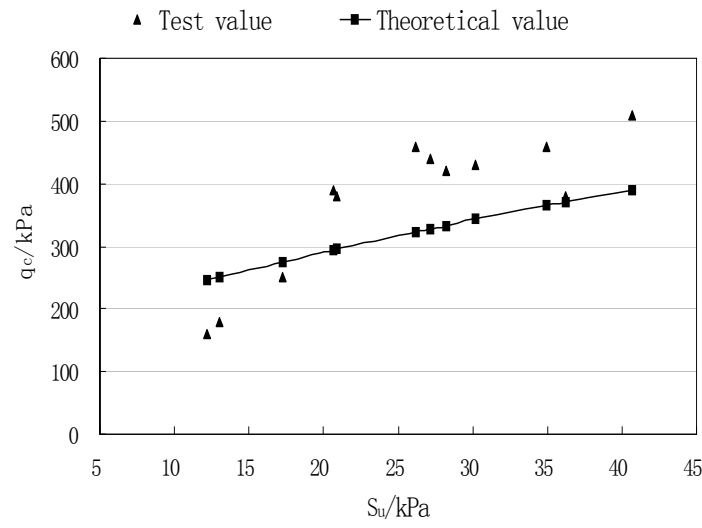

Fig. (5). Relation curve between $q_{c}$ with $S_{u}$.

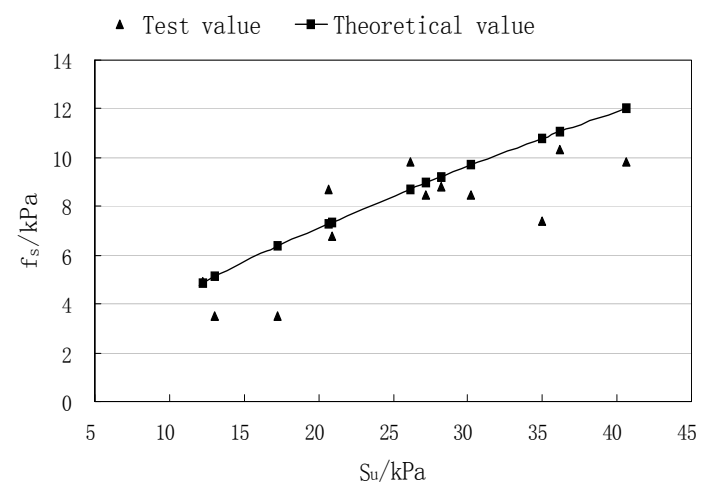

Fig. (6). Relation curve between $f_{s}$ with $S_{u}$.

the test value in a certain range $\left(\mathrm{c}_{\mathrm{u}}<40 \mathrm{kPa}\right)$, indicating that the theoretical formula is suitable for soft clay.

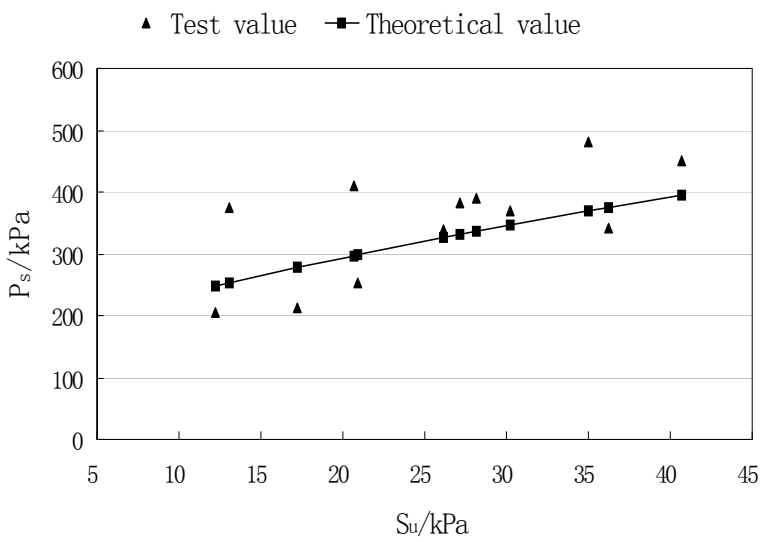

Fig. (7). Relation curve between $P_{s}$ with $S_{u}$.

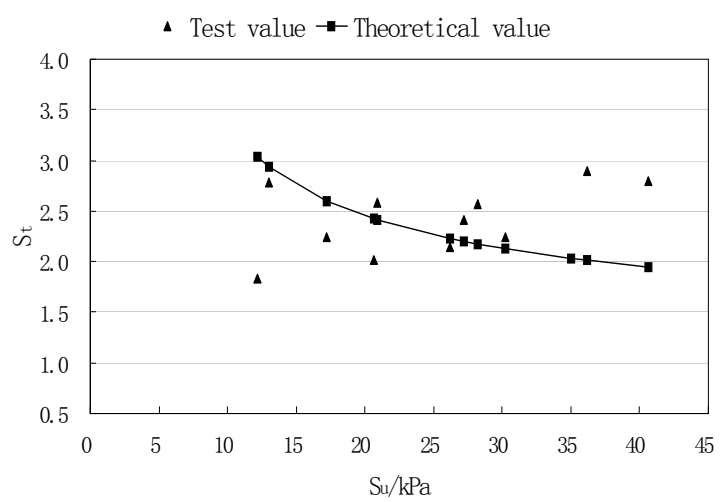

Fig. (8). Relation curve between $S_{t}$ with $S_{u}$.

(3) On the relationship between $P_{s}$ with $S_{u}$

Fig. (7) shows that the $P_{s} \sim S_{u}$ curve is a combination of the above two curves, $f_{s} \sim S_{u}$ and $q_{c} \sim S_{u}$, and in the same way, the theoretical value has a better consistency with the test value.

(4) On the relationship between $S_{t}$ with $S_{u}$

Fig. (8) shows that in theory, $S_{t}$ is reduced with the increase of $S_{u}$. However, the test data indicates the presence of one kind of soil, whose $S_{t}$ remains unchanged as soil shear strength increases. It is evident from the semi-empirical formula (10) based on the work of Schmertmann etc., only shows that $S_{t}$ would decrease with soil shear strength. The relation of $S_{t}$ with $S_{u}$ would be further developed.

Seen from the above comparison between the test and theoretical value, the two kinds of value have good consistency for saturated soft clay. If there is some deviation, the reason is the complex mechanical properties of soft clay, including: (1) The theory assumptions are simplified, such as the soil is assumed to be a simple elastic-plastic body; (2) The complex mechanical process in the penetration process is not taken into account, like the generation of pore water pressure (Tip resistance $q_{c}$ can be corrected as pore pressure is included), etc.; (3) The variability of soils properties, like the effect of residual shells in the soft soil formation, etc. 
The theoretical formula built the relationship among these important mechanical quantities of in situ tests, which not only makes huge amounts of test time and cost savings for the project design, but also provides a way for the further development of relative theory.

\section{CONCLUSION}

(1) The existed empirical relationship between the parameters of CPT and shear strength can be summed up as a general linear formula, in which the constant coefficient could be positive or negative.

(2) As a spatial-axisymmetric problem, with plasticity theory of cylindrical cavity expansion, the theoretical relationship between the parameters of CPT (including Tip resistance $q_{c}$, Side friction $f_{\mathrm{s}}$ and Specific penetration resistance $P_{s}$ ), the elastic parameters and cohesion of soil were established. Meanwhile, the relation between CPT parameters and shear strength value of vane test was also obtained.

(3) The above established theoretical relationship is nonlinear in terms of strength parameters, rather than linear expressed by the usual empirical formulae.

(4) Comparing results from the theoretical calculation with in situ data of CPT and VST in an actual ultra-soft soil (soft clay) treatment project in the Pearl River Delta indicated that good consistency between theoretical relations $\left(f_{s} \sim S_{u}, q_{c} \sim S_{u}\right.$ and $\left.P_{s} \sim S_{u}\right)$ and the test data. And semi-empirical formula on $S_{t}$ of VST only showed that sensitivity coefficient would decrease with soil shear strength, so the relation on sensitivity coefficient should be further developed.

(5) Seen form these derived theoretical relations, the penetration parameters can be directly determined by the conventional mechanical parameters (such as elastic parameters and cohesion) or vane shear parameters, and vice versa. Elasticity modulus E can also be calculated by tip resistance $q_{c}$ or side friction $f_{\mathrm{s}}$. The theoretical model can offer a very cost effective method for testing and can avoid some tests limited due to the site and/or other condition.

\section{CONFLICT OF INTEREST}

The authors confirm that this article content has no conflict of interest.

\section{ACKNOWLEDGEMENTS}

This work was supported by National Natural Science Foundation of China (Project No. 51178122).

\section{REFERENCES}

[1] Code for testing of building foundation. (DBJ 15-60-2008) . China Architecture \& Building Press, Beijing.

[2] X.Z. Chui, and H. Ding, "Approximate theoretical and experimental research progress on the head resistance of static cone penetration", Adv. Mech., vol. 34, no. 2, pp. 251-262, 2004.

[3] Z. Y. Lin, Test and Monitoring Handbook of Geotechnical Engineering. Shenyang: Liaoning Sci. \& Tech. Press, 1994.

[4] Z. M. Li, Soft soil foundation reinforcement with quality control. Beijing: China Architecture \& Building Press, 2011.

[5] P. K. Robertson, and R. G. Campanella, Guidelines for geotechnical design using $C P T$ and $C P T U$. Vancouver: University of British Columbia, 1988

[6] P. K. Robertson, and K. L. Cabal, Guide to cone penetration testing. California: Gregg Drilling \& Testing, Inc., 2012.

[7] S. W. Yan, X. W. Feng, J. F. Hou, and W. Li, "Deduction and application of strength parameters of soft clay by use of vane strength", Chinese J. Geotech. Eng., vol. 31, no. 12, pp. 1805-1810, 2009.

[8] J. H. Yang, and L. Xiong, "Analysis of correlation of situ-test results in the Yangtze Delta", Soil Eng., Found., vol. 24, no. 1, pp.79-80, 2010.

[9] Z. M. Li, Theory and engineering technology of ground improvement. Beijing: China Electric Power Press, 2014.

[10] X. N. Gong, Soil Plasticity. Hangzhou: Zhejiang University Press, 1999. 